\title{
The exposome in inflammatory bowel disease
}

\author{
Ashwin N Ananthakrishnan
}

\begin{abstract}
Division of Gastroenterology,

Inflammatory bowel disease (IBD) including Crohn's disease (CD) and ulcerative colitis (UC), Massachusetts General Hospital and Harvard Medical School, Boston, MA, USA

is an immunologically mediated chronic disease. The key underlying pathology is a deregulated immune response to commensal flora in a genetically susceptible host. Advances in genomics, epigenomics and understanding of the microbiome have brought forth the role of these spheres

Correspondence:

Dr. Ashwin N Ananthakrishnan

Email: aananthakrishnan@mgh. harvard.edu in the pathogenesis of IBD. Yet these factors explain only a small fraction of disease risk and our ability to predict relapses and response to treatment remains dismal. The external environment plays an important role in modifying the risk of IBD and in precipitating relapses in patients with established disease. The term 'exposome' was proposed to reflect a lifecourse of environmental influences beginning in-utero and proceeding right through childhood to adulthood. While the exposome is still a concept which needs practical perspective to enable better patient care, this review examines the gaps in our understanding that the IBD exposome helps explain. We further highlight the definition and parameters of this metric which can be incorporated for its application in research and clinical practice.
\end{abstract}

KEYWORDS: Crohn's disease, ulcerative colitis, environment, exposome

\section{Introduction}

The past two decades have witnessed significant advances in the field of inflammatory bowel disease (IBD) 'omics' research. Beginning with the initial understanding of familial contribution in IBD, to the recognition of the NOD2 gene ${ }^{1,2}$ and now 163 distinct predisposing genetic loci ${ }^{3}$ there has been a quantum leap in our understanding of IBD pathogenesis. The recent advancements in sequencing the gut microbiome have brought in perspective the role of the gut flora and the mucosal immune system in development of intestinal inflammation. ${ }^{4-6}$ However, despite these advances, several questions remain unanswered. Therapeutic advances have revolutionized our ability to achieve disease remission and reduced the need for surgeries and hospitalization. $^{7}$ And yet the cost and side effects of these therapies, including infections and malignancies, remain a major impediment. ${ }^{8,9}$ Even with the availability of new biologics, nearly
$40-50 \%$ of patients require dose escalation, and $10-20 \%$ of patients stop treatment every year. ${ }^{8,10}$. This review discusses the role of the 'exposome' as a concept with significant potential of practical clinical applications in IBD. However the recent advances in IBD genomics, epigenomics, microbiome and environmental risks are beyond the scope of this review and have been described in detail elsewhere..$^{5,11-15}$

\section{The Exposome}

The term exposome was coined in 2005 by Christopher Wild to encompass "life-course environmental exposures (including lifestyle factors), from the prenatal period onwards." ${ }^{16,17}$

With increasing knowledge about the role of the external and internal environment in IBD, the concept of exposome 
appears to offer a useful tool to predict risk, monitor diseasemodifying exposures, and enable early intervention to reduce risk and/or abate disease progression.

\section{The existing 'omes'}

A large international collaborative study recently described 163 distinct risk loci that modify the likelihood of Crohn's disease (CD) or ulcerative colitis (UC), ${ }^{3}$ expanding the genetic repertoire of IBD. ${ }^{18}$ Majority of these genes (110/163) are shared between UC and CD with similar effects. The elucidation of additional genetic loci also highlights a significant overlap of pathogenetic pathways between IBD and other autoimmune diseases, primary immunodeficiency states and mycobacterial infections. ${ }^{3}$ This new data has also brought into focus the role of the innate immune response, maintenance of intestinal barrier function, adaptive immune responses, endoplasmic reticulum stress, pathogen sensing, response to oxidative stress and epithelial restitution. ${ }^{12,13}$ Yet, genetics seem insufficient to explain several aspects of IBD epidemiology including the $<50 \%$ concordance rate in monozygotic twins, increased disease risk in immigrants from low prevalence country in a high prevalence country ${ }^{19,20}$, and the increasing incidence being noted in the West as well as in Asia. ${ }^{19,21,22}$ The cumulative disease variance explained is $<33 \%$ for both CD and UC with only modest effect sizes for most risk loci. ${ }^{3}$ Furthermore, these loci fail to reliably predict disease behavior and response to therapy. The NOD2 locus is associated with stricturing and penetrating disease, and ileal location of disease but is inconsistent in predicting response to therapy. ${ }^{23,24}$ Other risk genes encoding for autophagy (ATG16L1, IRGM) and interleukin-23 receptor (IL23R) also offer poor predictions of disease behavior. ${ }^{23}$ Thus, while genomics have contributed substantially in unraveling disease pathogenesis it is far from offering translational solutions for patient care.

The epigenome helps explain some gaps in the unexplained heritability of IBD ${ }^{11}$ but is still a nascent field in IBD. Agenomewide study by Nimmo et al examined the methylation status of 27,578 CpG sites across the genome in whole blood samples of 21 females with ileal CD and 19 controls. ${ }^{25}$ They identified 50 sites that demonstrated significantly different methylation between cases and controls. Some of these included site for dendritic cell activation, IL-17A mediated immune regulation, and defense against bacteria. Epigenetic changes also reflect environmental influences an individual has been exposed to right from in-utero to adulthood. Teh et al examined the methylome of 237 neonates and found that underlying genotype explained only $25 \%$ of the variably methylated regions. ${ }^{26}$ However, in-utero exposure to maternal smoking, maternal body mass index, maternal depression, birth weight, birth order and gestational age explained $75 \%$ of such variably methylated regions. Adult exposures such as smoking also induce epigenetic changes and may serve as markers for such environmental influences,${ }^{27}$ and in turn may influence and regulate metabolic traits. ${ }^{28}$

The third 'ome' influencing the pathogenesis of IBD is the gut microbiome. Patients with IBD have dysbiosis of the intestinal microbiome when compared to healthy controls. ${ }^{6}$ These changes are apparent even in new onset disease at the time of diagnosis. ${ }^{4}$ Microbiome changes in IBD include reduced diversity, decreased representation of 'protective bacteria' such as Firmicutes, and in subgroups such as ileal CD, an increase

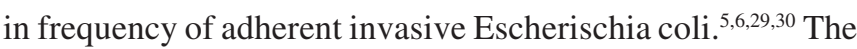
microbiome in IBD is influenced by source of sampling, concurrent medications, diet, smoking, and even age, and thus offers insight into the effect of external environment on the internal microenvironment. ${ }^{6}$

\section{The need for an exposome for IBD}

Despite the above advancements, several aspects of IBD remain unresolved. As seen above, genetics explains only a small fraction of disease variance. The epigenome and microbiome allow us to examine downstream changes in the host secondary to environmental exposures, but not all exposures translate into such effects. Furthermore, both the epigenome and the microbiome are affected by several factors. For example, the microbiome is influenced by long- and short-term dietary changes, and exposure to antibiotics. ${ }^{31-34}$ Such confounders make it difficult to accurately tease out remote and recent exposures from a mere analysis of the gut microbiome.

This brings us to the issue if existing tools are reliable in measuring the exposome? Initial studies used recall to assess past exposures including early life influences. While offering useful clues in support of the hygiene hypothesis, smoking and other behavioral risk factors, such retrospective studies are prone to recall bias and may overestimate the strength of association. Recent studies have used prospective cohorts such as the Nurses' Health Study cohort in the United States, and the European Prospective Study for Investigation into Cancer (EPIC) cohort, which have provided high-quality data in support of several key environmental risk factors for IBD. ${ }^{35-}$ 
${ }^{46}$ These include smoking, diet, medications, physical activity, vitamin D and psychosocial stress. ${ }^{35-47}$ While such questionnaire based studies offer considerable advantages, they are also limited in their ability to quantify the effect of specific exposures. Only a limited number of exposures at fixed time points can be assessed. There may be variation in the biological effect of environmental influences. For example, several genetic polymorphisms have been described that influence an individual's vitamin D status. ${ }^{48,49}$ Thus, while physical activity, dietary and supplemental vitamin D intake, race, and body mass index can provide an overall assessment of vitamin D status; the relative contribution of each of these variables, and in particular vitamin $\mathrm{D}$ synthesis in the skin from exposure to sunlight, which is the key determinant of vitamin D status, may vary based on underlying genotype. Similarly, area of residence and occupation may serve as proxies to exposure to air pollutants, but the biologic effect of air pollutants is determined by other factors like amount of time spent outdoors and leisure time physical activity, parameters that are difficult to capture consistently from questionnaires. Consequently, there is a significant need for the exposome, conceptualized as a comprehensive panel of environmental exposures ranging from air pollutants, chemical exposures, medications, diet to hormone and nutrient status, that will accurately quantify an individual's exposure to each of these factors over the course of their lifetime. We envision that this will not be biased by the mode of data acquisition, will represent exposures at biologically relevant sites, will not rely on individual recall, will be sensitive to changes over time and will not be influenced by the other variables being simultaneously measured (Table 1).

\section{Table 1: Characteristics of the ideal exposome}

- Measures a wide spectrum of exposures

- Quantifies biologically relevant exposure at the target site

- Not influenced by simultaneous measurement of other exposures

- Stable

- Sensitive to changes over time

- Provides a measure of both recent and remote exposure

\section{The Exposome - translating to utility}

What should the exposome comprise?

Much of the discussion around the exposome has centered around chemical exposures, ${ }^{16,17}$ lending itself to the nomenclature 'adductome', that may influence risk of cancer and other diseases. ${ }^{50}$ The association between chemical exposures and IBD has not been studied and consequently an exposome for IBD may have to inherently differ from an exposome for other diseases. Clearly, smoking and diet have a role in the pathogenesis of $\mathrm{CD}$ and $\mathrm{UC}$ and the exposome must include such exposures. Within diet, plasma levels may correlate with tissue levels of certain macro-nutrients but such a relationship may not hold true for all nutrients. Levels in the blood may not reflect a biologically relevant exposure. It is important for an exposome to include measures of both macroand micro-nutrients since both can be involved in the pathogenesis of IBD. It also remains to be seen whether such measures will be biased towards more recent or cumulative lifetime exposures. The association with air pollution is well recognized for cardiovascular disease but emerging evidence suggests an effect on gastrointestinal diseases including IBD as well. ${ }^{22,51,52}$ Thus some metric of environmental air and chemical pollutants would be imperative in the exposome. The role of infectious pathogens in the development of autoimmune diseases has also led to the concept of the 'infectome' or a measure of lifetime exposure to various infections. ${ }^{53}$ The association between specific infections and IBD risk has been weak and inconsistent, but bacterial and viral infections, both in infancy and adulthood have been proposed as risk factors for incident disease and relapses. ${ }^{54}$ Thus, an exposome would ideally include assessment of such exposures. Yet, one recognizes that there are a number of important environmental influences which as don't have any known biological signatures in the blood or tissue but have important influence on the disease. In particular, variables such as stress, anxiety, depression and sleep influence disease, relapses and response to treatment. ${ }^{39,54,55}$ However, quantifying such exposures remains a challenge. It may be inevitable that an exposome also requires incorporation of questionnaire based assessment of such psychometric exposures.

\section{Who should the exposome be measured in and when?}

The exposome would be of greatest benefit for predicting relapse. Conceptually the exposome could also influence therapeutic decisions and predict response to therapy, although environmental factors have not been noted for such influence so far. It is also plausible that the exposome may help identify individuals at risk like first degree relatives. Identifying detrimental environmental risk factors may allow one to change high-risk behaviors to help reduce subsequent risk of developing IBD. 
It also remains to be established whether such measurements should be performed cross-sectionally at a single time point, assessing all exposures up to that time, or longitudinally. The latter is preferable for some factors where recent exposure has greater relevance than remote. For example, in a study examining the association between depressive symptoms and IBD, we found a much stronger influence of recent depressive symptoms as compared to remote. ${ }^{39}$

\section{Is the Exposome independent of the genome?}

Current data suggests that susceptibility to environmental influences may depend on host genotype. For example, patients with single nucleotide polymorphisms near the CYP2A6/EGLN2 and the glutathione-S-transferase (GST) loci demonstrate stronger association between smoking and disease status (both CD and UC) when compared to individuals with the wild type. ${ }^{56}$ Similarly, the association between increased intake of $n-6 / n-3$ polyunsaturated fatty acids and $\mathrm{CD}$ is more prominent in individuals with specific variants at the CYP4F3 or FADS2 genes. ${ }^{57}$ Thus, it is likely that the exposome exerts its influence in context of the genome and that interpretation of one would require simultaneous assessment of the other. This would require large cohorts with sufficient power, and sophisticated computing tools to analyze the big data generated.

\section{The existing exposomes - where we are now?}

We already routinely obtain several measures of environmental exposure. Checking antibodies to hepatitis B and assessment of prior exposure to tuberculosis are already pre-requisites to starting biologic therapy and may be viewed as a part of the 'infectome'. Serological markers such as the anti-microbial antibody panels have shown promise in predicting disease complications, particularly in Crohn's disease and may even precede the development of IBD. ${ }^{58,59}$ Thus they may also represent one component of the exposome by measuring reactivity to bacterial or fungal antigens, and a measure of disruption of the intestinal barrier function and pathogen sensing.

There are several existing electronic applications or wearable devices that provide continuous assessments of exposures. Pedometers have long been available to track one's physical activity by measuring the distance walked or run. Wrist worn actigraphy has been an integral part of sleep research by measuring sleep duration, quality and disruption. Devices such as the Fitbit巴 combine sleep tracking, physical activity, diet, and other metrics and are becoming increasingly popular. Other wrist worn devices purport to infer mood by measuring skin conductance, skin temperature and physical motion. Modern technology is rapidly progressing towards an electronic exposome that will be able to measure several environmental attributes relevant to the natural history of IBD. Some of these measures can be collected passively while others such as diet may require more active participation. Integration of such data into the medical history of a patient will help examine the role of environmental factors. Electronic health record and novel tools like natural language processing provide accurate deep phenotyping of patients at a fraction of cost it takes to maintain large prospective cohorts. ${ }^{60,61}$

\section{The exposome - too much data?}

Adoption of a dynamic tool like the exposome demands some caution. The exposome may be more easily interpretable by patients than the genome, epigenome, microbiome or metabolome. While ready access to such data allows for early behavioral intervention, it is possible that such continued surveillance may also lead to anxiety and worsen the patient's quality of life. However, if the benefits of early intervention based on such data are significant, then routine monitoring of the exposome may be cost-effective. Another contention is who would have access to the exposome? Would that be part of one's medical records, and if so would other providers such as insurers have access to it? This leads to a debate similar to that pertaining genetic data - whether discrimination could be possible based on genetic (or exposure) data prior to actual development of the disease? Could someone face higher insurance costs solely based on their prior exposures rather than an actual disease state? While such concerns may be overshadowed by potential benefits, unintended adverse events need to be anticipated, studied and minimized.

\section{Conclusion}

Technological advances have significantly enhanced our understanding of IBD pathogenesis. Genomics and the gut microbiome have opened new insights into disease development and prediction of disease behavior. Yet many questions remain unanswered. Only a fraction of disease risk is predictable and relapses and response to treatment remain difficult to foresee. The exposome conceptually offers 
significant promise in resolving some of these challenges. There is a need to advance the exposome from concept to practical application, to provide an all-round understanding of IBD and help improve management of these patients.

\section{References}

1. Hugot JP, Chamaillard M, Zouali H, Lesage S, Cezard JP, Belaiche $\mathrm{J}$, et al. Association of NOD2 leucine-rich repeat variants with susceptibility to Crohn's disease. Nature. 2001;411:599-603.

2. Ogura Y, Bonen DK, Inohara N, Nicolae DL, Chen FF, Ramos R, et al. A frameshift mutation in NOD2 associated with susceptibility to Crohn's disease. Nature. 2001;411:603-6.

3. Jostins L, Ripke S, Weersma RK, Duerr RH, McGovern DP, Hui $\mathrm{KY}$, et al. Host-microbe interactions have shaped the genetic architecture of inflammatory bowel disease. Nature. 2012;491:119-24.

4. Gevers D, Kugathasan S, Denson LA, Vazquez-Baeza Y, Van Treuren W, Ren B, et al. The treatment-naive microbiome in new-onset Crohn's disease. Cell Host Microbe. 2014;15:382-92.

5. Kostic AD, Xavier RJ, Gevers D. The microbiome in inflammatory bowel disease: current status and the future ahead. Gastroenterology. 2014;146:1489-99.

6. Morgan XC, Tickle TL, Sokol H, Gevers D, Devaney KL, Ward DV, et al. Dysfunction of the intestinal microbiome in inflammatory bowel disease and treatment. Genome Biol. 2012;13:R79.

7. D'Haens GR, Panaccione R, Higgins PD, Vermeire S, Gassull M, Chowers Y, et al. The London Position Statement of the World Congress of Gastroenterology on Biological Therapy for IBD with the European Crohn's and Colitis Organization: when to start, when to stop, which drug to choose, and how to predict response? Am J Gastroenterol. 2011;106:199-212.

8. Van Assche G, Lewis JD, Lichtenstein GR, Loftus EV, Ouyang Q, Panes J, et al. The London position statement of the World Congress of Gastroenterology on Biological Therapy for IBD with the European Crohn's and Colitis Organisation: safety. Am J Gastroenterol. 2011;106:1594-602.

9. Afif W, Loftus EV, Jr. Safety profile of IBD therapeutics: infectious risks. Gastroenterol Clin North Am. 2009;38:691-709.

10. Gisbert JP, Panes J. Loss of response and requirement of infliximab dose intensification in Crohn's disease: a review. Am J Gastroenterol. 2009;104:760-7.

11. Ventham NT, Kennedy NA, Nimmo ER, Satsangi J. Beyond gene discovery in inflammatory bowel disease: the emerging role of epigenetics. Gastroenterology. 2013;145:293-308.

12. Khor B, Gardet A, Xavier RJ. Genetics and pathogenesis of inflammatory bowel disease. Nature. 2011;474:307-17.

13. Abraham C, Cho JH. Inflammatory bowel disease. N Engl J Med. 2009;361:2066-78.

14. Ananthakrishnan AN. Environmental risk factors for inflammatory bowel disease. Gastroenterol Hepatol (N Y). 2013;9:367-74.

15. Ananthakrishnan AN. Environmental triggers for inflammatory bowel disease. Curr Gastroenterol Rep. 2013;15:302.
16. Wild CP. Complementing the genome with an "exposome": the outstanding challenge of environmental exposure measurement in molecular epidemiology. Cancer Epidemiol Biomarkers Prev. 2005; 14:1847-50.

17. Wild CP. The exposome: from concept to utility. Int J Epidemiol. 2012;41:24-32.

18. Franke A, McGovern DP, Barrett JC, Wang K, Radford-Smith GL, Ahmad T, et al. Genome-wide meta-analysis increases to 71 the number of confirmed Crohn's disease susceptibility loci. Nat Genet. 2010;42:1118-25.

19. Cosnes J, Gower-Rousseau C, Seksik P, Cortot A. Epidemiology and natural history of inflammatory bowel diseases. Gastroenterology. 2011;140:1785-94.

20. Williams CN. Does the incidence of IBD increase when persons move from a low- to a high-risk area? Inflamm Bowel Dis. 2008;14 Suppl 2:S41-2.

21. Thia KT, Loftus EV, Jr., Sandborn WJ, Yang SK. An update on the epidemiology of inflammatory bowel disease in Asia. Am J Gastroenterol. 2008;103:3167-82.

22. Kaplan GG, Hubbard J, Korzenik J, Sands BE, Panaccione R, Ghosh S, et al. The inflammatory bowel diseases and ambient air pollution: a novel association. Am J Gastroenterol. 2010;105:2412-9.

23. Ananthakrishnan AN, Xavier RJ. How does genotype influence disease phenotype in inflammatory bowel disease? Inflamm Bowel Dis. 2013;19:2021-30.

24. Adler J, Rangwalla SC, Dwamena BA, Higgins PD. The prognostic power of the NOD2 genotype for complicated Crohn's disease: a meta-analysis. Am J Gastroenterol. 2011;106:699-712.

25. Nimmo ER, Prendergast JG, Aldhous MC, Kennedy NA, Henderson P, Drummond HE, et al. Genome-wide methylation profiling in Crohn's disease identifies altered epigenetic regulation of key host defense mechanisms including the Th17 pathway. Inflamm Bowel Dis. 2012;18:889-99.

26. Teh AL, Pan H, Chen L, Ong ML, Dogra S, Wong J, et al. The effect of genotype and in utero environment on interindividual variation in neonate DNA methylomes. Genome Res. 2014;24:1064-74.

27. Alegria-Torres JA, Baccarelli A, Bollati V. Epigenetics and lifestyle. Epigenomics. 2011;3:267-77.

28. Petersen AK, Zeilinger S, Kastenmuller G, Romisch-Margl W, Brugger M, Peters A, et al. Epigenetics meets metabolomics: an epigenome-wide association study with blood serum metabolic traits. Hum Mol Genet. 2014;23:534-45.

29. Darfeuille-Michaud A, Boudeau J, Bulois P, Neut C, Glasser AL, Barnich N, et al. High prevalence of adherent-invasive Escherichia coli associated with ileal mucosa in Crohn's disease. Gastroenterology. 2004;127:412-21.

30. Sokol H, Pigneur B, Watterlot L, Lakhdari O, Bermudez-Humaran LG, Gratadoux JJ, et al. Faecalibacterium prausnitzii is an antiinflammatory commensal bacterium identified by gut microbiota analysis of Crohn disease patients. Proc Natl Acad Sci U S A. 2008;105:16731-6.

31. Albenberg LG, Wu GD. Diet and the intestinal microbiome: associations, functions, and implications for health and disease. Gastroenterology. 2014;146:1564-72.

32. David LA, Maurice CF, Carmody RN, Gootenberg DB, Button 
JE, Wolfe BE, et al. Diet rapidly and reproducibly alters the human gut microbiome. Nature. 2014;505:559-63.

33. Wu GD, Chen J, Hoffmann C, Bittinger K, Chen YY, Keilbaugh $\mathrm{SA}$, et al. Linking long-term dietary patterns with gut microbial enterotypes. Science. 2011;334:105-8.

34. Ferrer M, Martins dos Santos VA, Ott SJ, Moya A. Gut microbiota disturbance during antibiotic therapy: a multi-omic approach. Gut Microbes. 2014;5:64-70.

35. Ananthakrishnan AN, Higuchi LM, Huang ES, Khalili H, Richter JM, Fuchs CS, et al. Aspirin, nonsteroidal anti-inflammatory drug use, and risk for Crohn disease and ulcerative colitis: a cohort study. Ann Intern Med. 2012;156:350-9.

36. Ananthakrishnan AN, Khalili H, De Silva PS, et al. Higher dietary fiber intake is associated with lower risk of Crohn's disease but not ulcerative colitis - a prospective study. Gastroenterology. 2012;Digestive Disease Week Abstract \# 863.

37. Ananthakrishnan AN, Khalili H, Higuchi LM, Bao Y, Korzenik JR, Giovannucci EL, et al. Higher predicted vitamin D status is associated with reduced risk of Crohn's disease. Gastroenterology. 2012;142:482-9.

38. Ananthakrishnan AN, Khalili H, Konijeti GG, Higuchi LM, de Silva P, Fuchs CS, et al. Long-term intake of dietary fat and risk of ulcerative colitis and Crohn's disease. Gut. 2014;63:776-84.

39. Ananthakrishnan AN, Khalili H, Pan A, Higuchi LM, de Silva P, Richter JM, et al. Association between depressive symptoms and incidence of Crohn's disease and ulcerative colitis: results from the Nurses' Health Study. Clin Gastroenterol Hepatol. 2013;11:57-62.

40. Higuchi LM, Khalili H, Chan AT, Richter JM, Bousvaros A, Fuchs CS. A prospective study of cigarette smoking and the risk of inflammatory bowel disease in women. Am J Gastroenterol. 2012;107:1399-406.

41. Khalili H, Higuchi LM, Ananthakrishnan AN, Manson JE, Feskanich D, Richter JM, et al. Hormone therapy increases risk of ulcerative colitis but not Crohn's disease. Gastroenterology. 2012;143:1199-206.

42. Khalili H, Higuchi LM, Ananthakrishnan AN, et al. Oral contraceptives, reproductive factors, and risk of inflammatory bowel disease. Digestive Disease Week. 2012.

43. Khalili H, Huang ES, Ananthakrishnan AN, Higuchi L, Richter JM, Fuchs CS, et al. Geographical variation and incidence of inflammatory bowel disease among US women. Gut. 2012;61:1686-92.

44. Chan SS, Luben R, Bergmann MM, Boeing H, Olsen A, Tjonneland A, et al. Aspirin in the aetiology of Crohn's disease and ulcerative colitis: a European prospective cohort study. Aliment Pharmacol Ther. 2011;34:649-55.

45. Hart AR, Luben R, Olsen A, Tjonneland A, Linseisen J, Nagel G, et al. Diet in the aetiology of ulcerative colitis: a European prospective cohort study. Digestion. 2008;77:57-64.

46. John S, Luben R, Shrestha SS, Welch A, Khaw KT, Hart AR. Dietary n-3 polyunsaturated fatty acids and the aetiology of ulcerative colitis: a UK prospective cohort study. Eur $J$ Gastroenterol Hepatol. 2010;22:602-6.
47. Khalili H, Ananthakrishnan AN, Konijeti GG, Liao X, Higuchi LM, Fuchs CS, et al. Physical activity and risk of inflammatory bowel disease: prospective study from the Nurses' Health Study cohorts. BMJ. 2013;347:f6633.

48. Ahn J, Yu K, Stolzenberg-Solomon R, Simon KC, McCullough ML, Gallicchio L, et al. Genome-wide association study of circulating vitamin D levels. Hum Mol Genet. 2010;19:2739-45.

49. Wang TJ, Zhang F, Richards JB, Kestenbaum B, van Meurs JB, Berry D, et al. Common genetic determinants of vitamin D insufficiency: a genome-wide association study. Lancet. 2010;376:180-8.

50. Kanaly RA, Hanaoka T, Sugimura H, Toda H, Matsui S, Matsuda T. Development of the adductome approach to detect DNA damage in humans. Antioxid Redox Signal. 2006;8:993-1001.

51. Ananthakrishnan AN, McGinley EL, Binion DG, Saeian K. Ambient air pollution correlates with hospitalizations for inflammatory bowel disease: an ecologic analysis. Inflamm Bowel Dis. 2011;17:1138-45.

52. Beamish LA, Osornio-Vargas AR, Wine E. Air pollution: An environmental factor contributing to intestinal disease. J Crohns Colitis. 2011;5:279-86.

53. Bogdanos DP, Smyk DS, Invernizzi P, Rigopoulou EI, Blank M, Pouria S, et al. Infectome: a platform to trace infectious triggers of autoimmunity. Autoimmun Rev. 2013;12:726-40.

54. Singh S, Graff LA, Bernstein CN. Do NSAIDs, antibiotics, infections, or stress trigger flares in IBD? Am J Gastroenterol. 2009;104:1298-313.

55. Bernstein CN, Singh S, Graff LA, Walker JR, Miller N, Cheang M. A prospective population-based study of triggers of symptomatic flares in IBD. Am $J$ Gastroenterol. 2010;105:1994-2002.

56. Ananthakrishnan AN, Nguyen DD, Sauk J, Yajnik V, Xavier RJ. Genetic polymorphisms in metabolizing enzymes modifying the association between smoking and inflammatory bowel diseases. Inflamm Bowel Dis. 2014;20:783-9.

57. Costea I, Mack DR, Lemaitre RN, Israel D, Marcil V, Ahmad A, et al. Interactions between the dietary polyunsaturated fatty acid ratio and genetic factors determine susceptibility to pediatric Crohn's disease. Gastroenterology. 2014;146:929-31.

58. Israeli E, Grotto I, Gilburd B, Balicer RD, Goldin E, Wiik A, et al. Anti-Saccharomyces cerevisiae and antineutrophil cytoplasmic antibodies as predictors of inflammatory bowel disease. Gut. 2005;54:1232-6.

59. Iskandar HN, Ciorba MA. Biomarkers in inflammatory bowel disease: current practices and recent advances. Transl Res. 2012;159:313-25.

60. Mandl KD, Kohane IS. Escaping the EHR trap-the future of health IT. N Engl J Med. 2012;366:2240-2.

61. Ananthakrishnan AN, Cai T, Savova G, Cheng SC, Chen P, Perez $\mathrm{RG}$, et al. Improving case definition of Crohn's disease and ulcerative colitis in electronic medical records using natural language processing: a novel informatics approach. Inflamm Bowel Dis. 2013;19:1411-20. 